\title{
Linear Zosteriform Lichen Planus Pigmentosus: A Single Case Report
} \author{
Caldeira Bastos ${ }^{2}$ and Gustavo Moreira Amorim ${ }^{1,3^{*}}$ \\ ${ }^{1}$ Universidade do Sul de Santa Catarina - UNISUL, Brazil \\ ${ }^{2}$ Instituto Diagnóstico De Anatomia Patológica - IDAP, Brazil \\ ${ }^{3}$ Federal University of Rio de Janeiro - UFRJ, Rio de Janeiro, Brazil
}

Luisa Krusser Vanin ${ }^{1}$, Celyna Scariot Grezzana ${ }^{1}$, Laura Gomes Da Broi ${ }^{1}$, Gabriel Amorim ${ }^{1}$, José

Received: April 17, 2018; Accepted: May 09, 2018 ; Published: May 18, 2018

*Corresponding author: Gustavo Moreira Amorim, Federal University of Rio de Janeiro, Rio de Janeiro, Brazil, Tel: +55 2139382580 ; E-mail: gustavomoreiraamorim@hotmail.com

\section{Abstract}

We reported a case of a female patient, 27 years of age, attended due to an acquired dyschromia, whose clinical and pathological diagnosis was of Lichen Planus Pigmentosus (LPP). Facing the clinic reported, with the linear pattern of the lesions, restricted to the area of one sensitive dermatome, we classified the conditions as a linear zosteriform LPP. The literature was consulted and presented with the clinical report, in order to draw attention to this rare entity. The patient is still under follow-up.

Mesh Terms: Pigmentation disorder; Lichen planus; Lichen planus pigmentosus;

\section{Background}

Lichen planus pigmentosus is a rare acquired dyschromia that occurs with the appearance of hyperchromic lesions in the skin, and rarely, in the mucosa [1]. Although the description of an acquired hyperchromic dermatosis was previously reported, the name and description of LPP as a specific entity is attributed to the report made in India by Bhutani, et al. in 1974 [2].

It is an uncommon disease, however incidence or prevalence are unknown. It manly affects young adults, between the third and fifth decades of life. It predominates in patients with higher phototypes, especially those with Fitzpatrick IV or more. A female predominance is reported, with the exception of one study in which men with hepatitis C were more affected [3-6].

The etiopathogenesis of LPP is similar to lichen planus, where there is a change in cellular immunity mediated by cytotoxic $\mathrm{T}$ lymphocytes. Those lymphocytes recognize and direct their cytotoxic action against keratinocytes, which determines interface dermatitis and vacuolization of the basal layer, culminating with intense pigmentary incontinence. Although, unlike lichen planus, this lymphocytic infiltrate acts rapidly and intensely, being sufficient to damage the dermo-epidermal junction, without causing an epidermal reaction of acanthosis and hypergranulosis. The infiltrate soon disperses, leaving only the melanophages in the papillary dermis. Due to that, LPP is understood, by some authors, as an abortive classic form lichen planus $[3,4,7]$.

Some etiopathogenic hypotheses were proposed. Topical use and Consumption of mustard oil, use of amla oil (typical fruit of India whose oil is used in hair treatments), henna tincture, hair dyes, cold cream vehicle, also environmental pollution $[3,7]$. Ultraviolet radiation sun exposure is understood as a triggering or exacerbating factor, since there is a clear predominance of the lesions on sun-exposed areas [4,8]. Finally, as previously mentioned, the possible relationship with the hepatitis $\mathrm{C}$ virus [6].

Clinically the disease is manifested by small oval-shaped macules, with poorly delimited borders, hyperchromic, initially light brown, evolving to a blackish-brown to grayish [3]. It typically affects the face and neck, has insidious beginning, and a slowly progressive evolution [4].

Usually they are asymptomatic, however some patients may complain of mild pruritus. The pruritus could help in the identification of the active phase of the disease. Rarely, patients may also refer to a burning pain sensation. The classic presentation is usually bilateral and symmetrical arrangement of the lesions $[3,5,7]$.

Known clinical variants of the classical LPP are the inverted or inverse LPP, and the linear LPP [4]. Inverted LPP was described in 2001 by Pock et al., and was defined as the LPP variant limited to flexural areas, both non-sun-exposed areas [9]. The linear subtype, as the name suggests, presents as a characteristic the peculiar distribution of the lesions, aligned, describing a path, being able to follow the lines of Blaschko (LPP linear Blasckoide) uni or bilateral, following one or more Blaschko lines; or in a sensory dermatome restricted form, usually in the thorax, therefore in herpes zoster-like topography (linear zosteriform LPP) $[8,10]$.

We presented a case of linear zosteriform LPP, documented with photographs and histopathology, in order to draw attention 
to the rare acquired dyschromia, comparing the present findings with what is described in the literature.

\section{Case Report}

A 27-year-old female patient presented to us with asymptomatic hyperchromic macules on her skin, for about 15 months. The lesions appeared in the right lumbar region, with an increase in size and number of lesions in the first month after the beginning, reaching the region of the right abdominal flank, soon stabilizing and remaining without significant evolution. She denied that the lesions, at the onset or even along the evolution, had presented erythema or edema. She denied pruritus. She also denied local trauma before the onset of the lesions.

It is a healthy patient, who denied use of any type of medication.

At the examination, multiple lenticular, hyperchromic macules of light and dark brown staining, non-confluent, with different sizes, in a linear arrangement, identified in the right paravertebral region, extending to the right abdominal flank. There was no

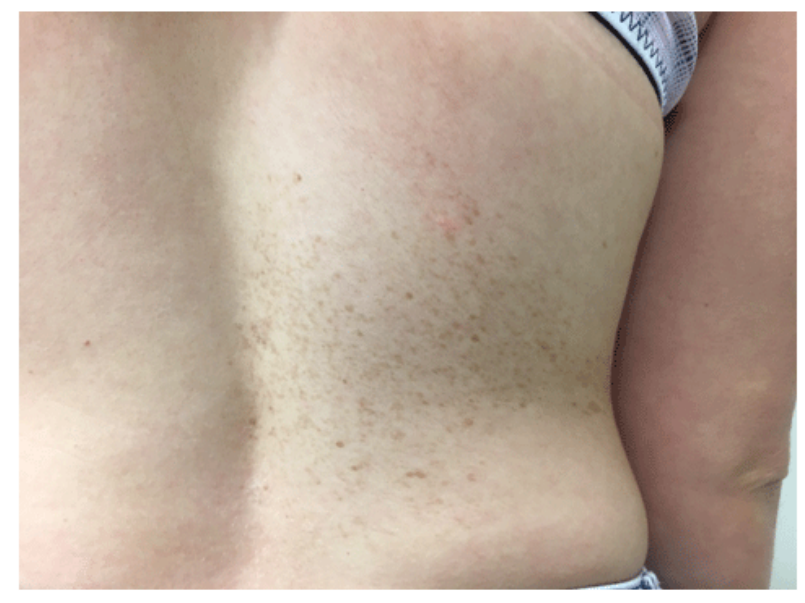

Figure 1: Multiple lenticular, hyperchromic macules of light and dark brown staining, non-confluent, with different sizes

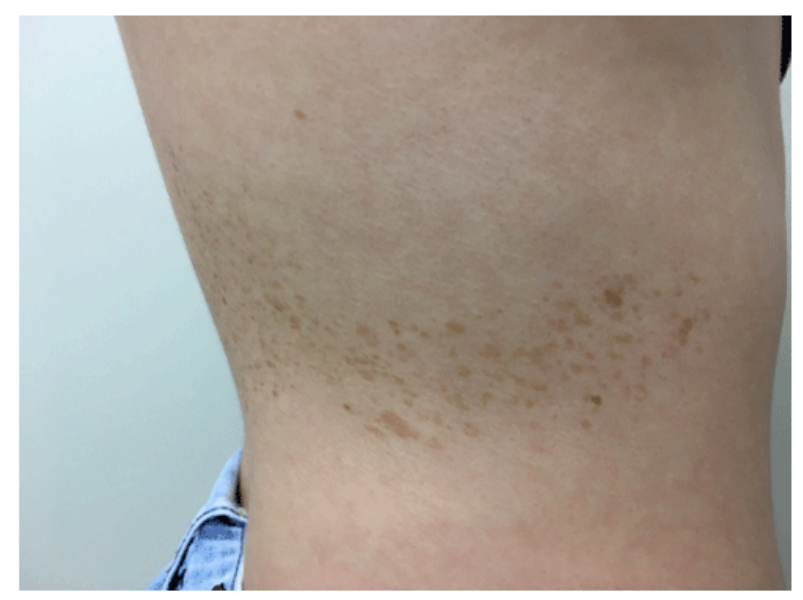

Figure 2: Approximate photo showing the linear aspect, with the disposition of the lesions restricted to one sensory dermatome

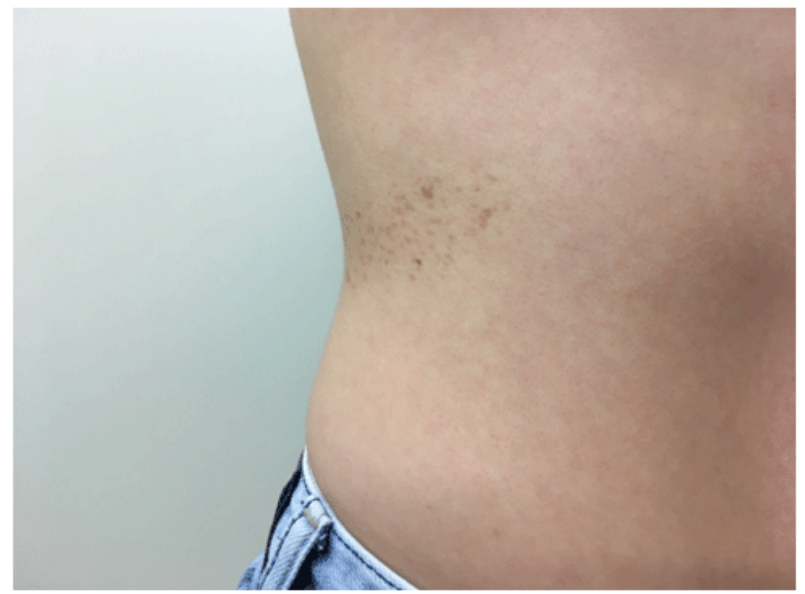

Figure 3: Extension of lesions to the abdominal flank, maintaining a linear aspect

infiltration during palpation (Figures 1, 2 and 3). The scalp, oral cavity, extremities and nails were actively evaluated, without any alteration.

An incisional biopsy of one of the lesions was performed, where the histopathological examination revealed a perivascular mononuclear infiltrate in the superficial dermis, besides pigmentary incontinence, identified by a significant number of melanophages in the papillary dermis. The staining by Fontana Masson confirmed corroborated with the impression of the pigmentary incontinence. (Figures 4 and 5).

Diagnosis of LPP was made, in its linear zosteriform form. Subsequent to the diagnosis, a history of contact with the triggers suggested in the literature was questioned and reject by the patient. Hepatitis C serology was negative.

We started treatment with topical tacrolimus, $0,1 \%$, ointment, once a day and $10 \%$ glycolic acid with $4 \%$ hydroquinone cream, once a day, at night. Patient is still being followed.

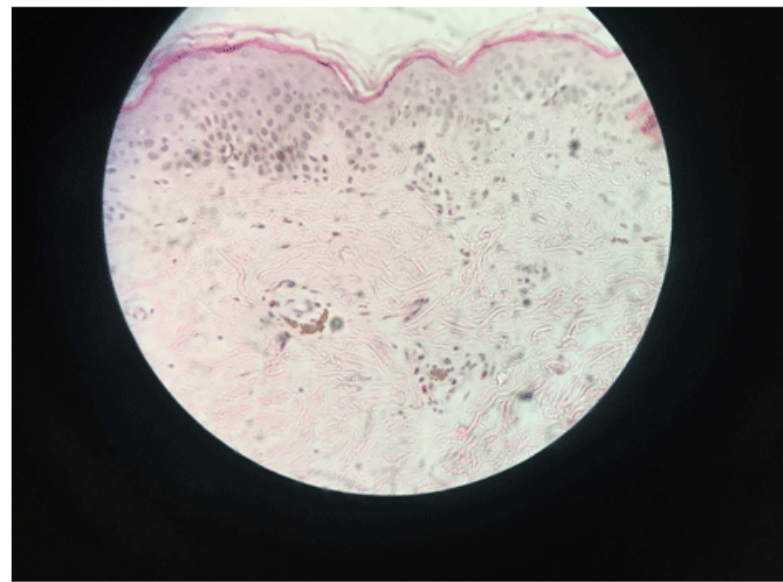

Figure 4: HE, 400X. Pigmentary incontinence. Significant number of melanophages 


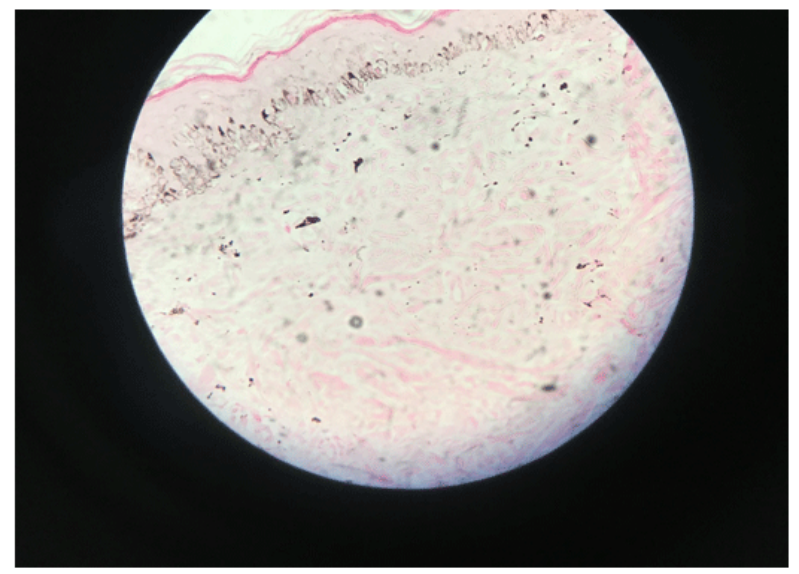

Figure 5: Fontana-Masson, 400x. Confirming pigmentary incontinence by identifying melanophages

Patient signed a formal consent allowing us to publish her clinical pictures. The authors committed themselves to the confidentiality of the data.

\section{Conclusions}

Linear zosteriform LPP represents a rare subtype of LPP $[10,11]$.

The differential diagnosis should include other dermatoses. Initially, due to the lenticular aspect of the lesions, besides the dark brown color, lentiginose agminata was a hypothesis, but histopathology, which would show an increase in the number of normal melanocytes along the dermo-epidermal junction, in addition to the increase of melanin deposition in the keratinocytes baseline, excluded this diagnosis [12].

With the histopathological pattern demonstrating pigment incontinence, a relevant differential diagnoses is fixed drug eruption, on final stages, non-inflammatory, as well as Ashy dermatosis, also known as persistent dyschromic erythema. Both dermatoses can be ruled out based on clinical history and dermatological examination.

Fixed pigmented erythema or fixed drug eruption is a common drug-related cutaneous reaction, typically caused by non-steroidal anti-inflammatory drugs and antibiotics. It is characterized by the sudden appearance of multiple erythematous-purplish macules and spots, some of them becoming erythematous-edematous, forming plaques, with well-demarcated borders and being able to evolve with bubble formation in the central region. They usually initiate within 48 hours of the ingestion of the related medication and slowly involve into residual-aspect hyperpigmented macules. With the re-exposure to the medication, the old lesions start to present a new inflammatory phase, along with new acute lesions appear as well [13]. Although the chronic stage can be justified by the histopathology, there is no clinical history of the inflammatory phase, either at the beginning or over the 15 months, nor an apparent relationship with drugs.

Ashy dermatosis or Erythema Dyschromicum Perstans
(EDP) is an acquired idiopathic dyschromia, characterized by the appearance of multiple bluish-gray macules, with slightly erythematous borders in young adults in the third decade of life, seeming to predominate in females. The lesions typically affect the trunk, in a diffuse, symmetrical and bilateral manner. Progressively, the lesions are confluent, forming larger spots. EDP is a relevant differential diagnosis of classical LPP. In the present case, by the pattern of lenticular, non-confluent lesions, with unilateral linear distribution, EDP can be discarded [14].

Treatment of LPP is difficult. There is no robust evidence for first-line treatment recommendation [1-4]. High potency topical corticosteroids, calcineurin inhibitors and hydroquinone-based depigmenting agents and alpha hydroxy acids are described as options. Among the options, tacrolimus was tested in an open, non-randomized, clinical trial, where a positive result was demonstrated in $53,8 \%$ of the patients [6]. From the systemic point of view, isolated reports of the use of prednisone, dapsone and isotretinoin, the latter being tested at a low dose for patients with classical LPP, with some response [5]. Finally, some reports of laser strategies [1].

We sought, with this illustrative case, to draw attention to this rare entity, in addition to discussing the main differential diagnosis.

\section{References}

1. Ghosh A, Coondon A. Lichen planus pigmentosus: the controversial consensus. Indian J Dermatol. 2016;61(5):482-486. doi: 10.4103/0019-5154.190108

2. Bhutani LK, Bedi TR, Pandhi RK, Nayak NC. Lichen planus pigmentosus. Dermatologica. 1974;149(1):43-50

3. Kanwar AJ, Dogra S, Handa S, Parsad D, Radotra BD. A study of 124 Indian patients with lichen planus pigmentosus. Clin Exp Dermatol. 2003;28(5):481-485.

4. Robles-Méndez JC, Rizo-Frías P, Herz-Ruelas ME, Pandya AG, Ocampo Candiani J. Lichen planus pigmentosus and its variants: review and update. Int J Dermatol. 2018;57(5):505-514. doi: 10.1111/ijd.13806

5. Muthu SK, Narang T, Saikia UN, Kanwar AJ, Parsad D, Dogra S. Lowdose oral isotretinoin therapy in lichen planus pigmentosus: an open-label non-randomized prospective pilot study. Int J Dermatol. 2016;55(9):1048-1054. doi: 10.1111/ijd.13293

6. Al-Mutairi N, El-Khalawany M. Clinicopathological characteristics of lichen planus pigmentosus and its response to tacrolimus ointment: an open label, non-randomized, prospective study. J Eur Acad Dermatol Venereol. 2010;24(5):535-540. doi: 10.1111/j.14683083.2009.03460.x

7. Bhat RM, Mathanda TR, Jayaprakash CS, Dandakeri S. Clinical, Histopathological Characteristics and Immunohistochemical Findings in Lichen Planus Pigmentosus. Indian J Dermatol. 2017;62(6):612617. doi: 10.4103/ijd.IJD_148_17

8. Polat M, Tuman B, Özyalvaçlı G. A case of unilateral blaschkoid lichen planus pigmentosus. An Bras Dermatol. 2017;92(3):443-443. doi: 10.1590/abd1806-4841.20175664

9. Pock L, Jelínková L, Drlík L, Abrhámová S, Voitechovská S, Sezemská D, et al. Lichen planus pigmentosus-inversus. J Eur Acad Dermatol Venereol. 2001;15(5):452-454. 
10. Cho S, Whang KK. Lichen planus pigmentosus presenting in zosteriform pattern. J Dermatol. 1997;24(3):193-197.

11. Vineet R, Sumit S, K GV, Nita k. Lichen planus pigmentosus um linear and xosteriforms pattern along the lines of Blaschko. Dermatol Online J. 2015;21(10).

12. Dawoud NM, Bakry OA, Dawoud MM, Samaka RM. Agminated lentiginosis or segmental neurofibromatosis: A diagnostic challenge. Indian J Dermatol Venerel Leprol. 2016;82(1):75-77. doi: 10.4103/0378-6323.171651
13. Heng YK, Yew YW, Lim DS, Lim YL. An update of fixed drug eruption in Singapore. J Eur Acad Dermatol Venereol. 2015;29(8):1539-1544. doi: $10.1111 /$ jdv.12919

14. Chang SE, Kim HW, Shin JM, Lee JH, Na JL, Roh MR, et al. Clinical and histological aspecto $\mathrm{f}$ erythema dyschromicum perstans in Korea: a review of 68 cases. J Dermatol. 2015;42(11):1053-1057. doi: $10.1111 / 1346-8138.13002$ 